\title{
Postenucleation orbital radiotherapy for the treatment of malignant melanoma of the choroid with extrascleral extension
}

\author{
P G Hykin, A C E McCartney, P N Plowman, J L Hungerford
}

\begin{abstract}
The outcome is reported in 17 patients in whom an eye was enucleated for malignant melanoma of the choroid with extrascleral extension and who subsequently underwent adjuvant external beam radiotherapy to the orbit as the primary treatment of the extraocular spread of their tumour. Extrascleral extension was encapsulated in five, nonencapsulated in two, and had been surgically transected at enucleation in 10 cases. All the patients have been followed up from enucleation to the present day. Orbital recurrence occurred in only one patient. The overall actuarial survival rate was $51 \%$ at 5 years, $\mathbf{4 4 \%}$ at 10 , and $33 \%$ at 15 years. A low orbital recurrence rate of $6 \%$ compares very favourably with published figures for this event after enucleation for melanoma with extrascleral extension but without radiotherapy. Adjuvant orbital radiotherapy may have a place in the treatment of selected cases of extracleral extension of intraocular malignant melanoma.
\end{abstract}

The incidence of extrascleral extension of choroidal malignant melanoma has been reported to be between 8 and $40 \% \cdot{ }^{1-7}$ Extraocular spread carries a poor prognosis for survival, with an overall mortality rate from melanoma metastases of between 66 and $73 \%$ compared with $39 \%$ without extrascleral extension. ${ }^{13}$ There is agreement that massive extraocular spread should be treated by orbital exenteration, ${ }^{1378}$ but the management of more moderate degrees of extraocular extension of uveal melanoma is controversial.

At Moorfields Eye Hospital and at Saint Bartholomew's Hospital exenteration of the orbit is advised following enucleation for a posterior melanoma with extrascleral extension only when extensive residual tumour is seen in the orbit during the operation. Exenteration is not recommended when an extrascleral nodule of melanoma is seen at operation but is apparently removed complete with the eye. This applies whether the nodule is reported as being encapsulated, non-encapsulated, or transected on subsequent histopathological examination. Instead, adjuvant orbital radiotherapy is advised. No additional therapy is recommended for melanoma invading the sclera or scleral channels or confined to the episclera.

Few ocular oncology centres advocate postenucleation radiotherapy for extrascleral extension of melanoma and little has been published on the results of its use. The object of this report is to present the results of external beam radio- therapy in the treatment of extraocular extension in terms both of local recurrence and of survival and to determine if it has a role in the future management of selected cases.

\section{Patients and methods}

The case notes were reviewed of all patients with extraocular extension of choroidal malignant melanoma treated in the Ocular Oncology Clinics at Moorfields Eye Hospital and St Bartholomew's Hospital and who had received orbital radiotherapy. Patients who had had external beam radiotherapy prior to enucleation or after orbital exenteration were excluded, as were patients with melanoma of the iris or ciliary body and patients with less than five years' follow-up. Between 1964 and 198220 consecutive cases fulfilled the criteria for inclusion in the

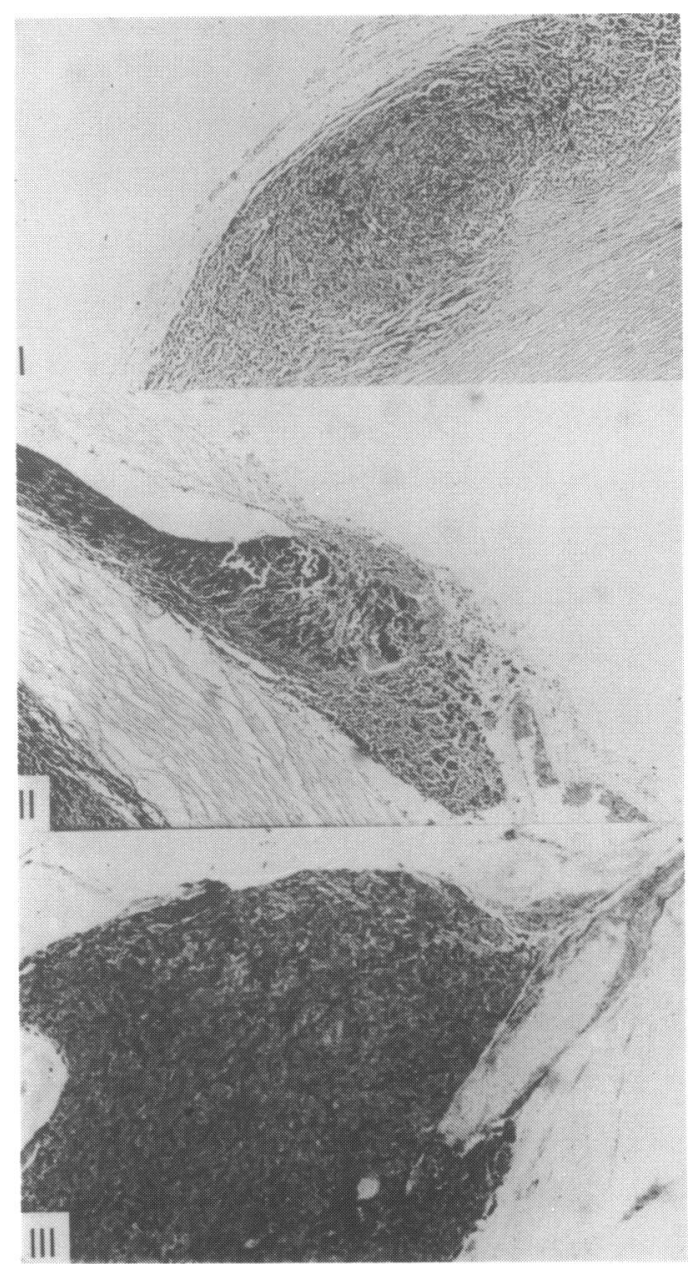

Figure 1: Histological sections $(\times 47)$ of enucleated eye and orbital tissues illustrating: (1) nodular encapsulated, (2) nodular non-encapsulated, and (3) surgically transected extrascleral extension. 
study, though sufficient follow-up data were not obtained in three. The histology was reviewed in each of the remaining 17 cases, but the data were not analysed for cytological criteria of malignancy. The maximum diameter of the tumour and of the extrascleral deposit was measured. The extrascleral deposit was reported as (1) nodular encapsulated (that is, undisturbed by enucleation), (2) nodular non-encapsulated (not obviously transected, but not encapsulated), or (3) surgically transected (Fig 1).

External beam radiotherapy was given within two months of enucleation. Of 16 patients receiving megavoltage photons, 14 were treated with cobalt-60 and two with caesium-137. One patient was treated with megavoltage electrons. The average prescribed dose was $50 \mathrm{~Gy}$ (range 35-60 Gy) in 22 fractions (range 7-33) over a mean of 35 days (range 15-52). All the patients were followed up until the time of death or until the present day, the minimum follow-up being five years. All who are alive have been seen in the last nine months and have had a general and an orbital examination. Gamma glutamyl transferase, liver ultrasound, and bone scan examinations were performed if clinically indicated. Information on deceased patients was obtained from case notes, death certificates, and the United Kingdom Cancer Register. Not all dead patients underwent post-mortem examination. Results were analysed by life table construction and calculation of actuarial survival, and the statistical significance was estimated by Fisher's exact probability test.

\section{Results}

There were 12 male and five female patients. The average age at enucleation was $58 \cdot 2$ years (range 31-71). The histology was spindle cell melanoma in 13 cases and mixed spindle and epithelioid in four. The type of extrascleral extension was nodular encapsulated in five, non-encapsulated in two, and transected in 10 cases. The mean largest diameter of the intraocular tumour was $13.5 \mathrm{~mm}$ (range $8-19 \mathrm{~mm}$ ) and the mean largest diameter of the extrascleral deposit was $3.1 \mathrm{~mm}$ (range $2-10.5 \mathrm{~mm}$ ).

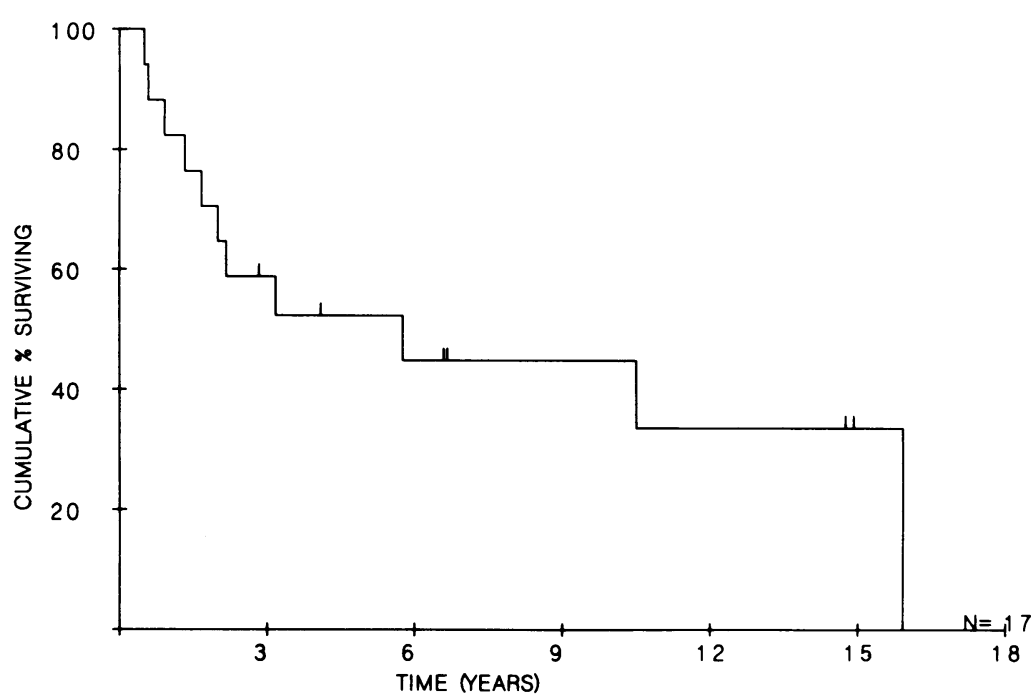

Figure 2: Actuarial survival curve (17 cases) after enucleation and orbital radiotherapy for malignant melanoma of the choroid with extrascleral extension.
SURVIVAL

Fourteen of the 17 patients have died. Eleven died from malignant melanoma metastases, two from cardiorespiratory failure, and one from a primary lung tumour. Three patients are alive and were free of disease when last seen. The actuarial overall survival rate was $51 \%$ at 5 years, $44 \%$ at 10 years, and $33 \%$ at 15 years (Fig 2). There was no significant difference in the survival of patients with transected versus nontransected extraocular spread at 5 and 15 years $(p>0.5)$. In addition, in this study the maximum tumour diameter $(>10$ and $\leqslant 10 \mathrm{~mm})$ and the size of the extraocular spread ( $>5$ and $\leqslant 5 \mathrm{~mm}$ ) had no statistically significant bearing on survival $(\mathrm{p}>0.5)$.

\section{ORBITAL RECURRENCE}

One patient developed orbital recurrence. He had undergone enucleation of the right eye at age 66 years, four months after diagnosis of a choroidal melanoma. The tumour was of spindle cell type, and an extrascleral extension was surgically transected at operation. The maximum tumour diameter was $13 \mathrm{~mm}$ and maximum diameter of extraocular extension $3.75 \mathrm{~mm} .60$ Gy megavoltage photons were given in 30 fractions within 50 days of enucleation. Orbital recurrence occurred three weeks after radiotherapy was completed. He then underwent early orbital exenteration but died of disseminated melanoma 20 months later.

\section{Discussion}

Extrascleral extension of choroidal melanoma has a highly significant adverse effect on local tumour control. Starr and Zimmerman observed that there was a 26 times greater likelihood of orbital recurrence with extrascleral extension than without.' Orbital recurrence was six times more likely to occur if the tumour nodule was non-encapsulated or surgically transected than if it was encapsulated, and $50 \%$ of tumours without a capsule or apparently transected will recur in the orbit.'

There may not be an independent adverse effect of extrascleral extension on survival rate. Starr and Zimmerman found that local extraocular spread reduces the five-year survival rate from $67 \%$ without this finding to $34 \%$ with, ${ }^{\prime}$ but in a multivariate analysis Kidd and coworkers were unable to demonstrate a significant independent adverse effect of extrascleral extension of melanoma on the prognosis for survival. ${ }^{9}$ Starr and Zimmerman had already demonstrated that there was a significantly greater number of melanomas of more malignant cell type among patients with extrascleral extension than among those without,' and this feature of tumours which extend out of the eye locally may account for the increased mortality with which they are associated.

There are no data from multivariate analysis to establish whether the development of orbital recurrence has an independent adverse effect on survival rate. However, the five-year survival rate has been found to be reduced from $34 \%$ for all patients with extrascleral extension to $11 \%$ for 
those who subsequently have a recurrence in the orbit,' and there would seem to be strong grounds for attempting to prevent the development of orbital recurrence by adjuvant orbital radiotherapy.

The primary aim of any treatment proposed for extrascleral extension of choroidal malignant melanoma is therefore to intercept and remove or destroy any malignant cells which may be in transit through the orbit to distant sites and thereby to prevent metastasis if this has not already taken place. A subsidiary aim is to prevent orbital recurrence and to limit the chance of this in turn leading to metastatic death. The optimal method for achieving these ends is controversial, as are the relative benefits to the patient, but the recommended approach now depends largely on the extent of the extraocular element of the tumour.

Orbital exenteration has been the standard treatment for extrascleral extension in many centres, and its role continues under discussion. Although Rendahl maintained that exenteration was never indicated for extrascleral extension of melanoma because it did not improve the outlook for survival, ${ }^{7}$ Shammas and Blodi reported a high five-year survival rate following the operation and considered that the procedure may be curative if performed early. ${ }^{3}$ More recently, however, Kersten, with Blodi and others, observed that after extended follow-up the tumour-associated mortality rate approached that of patients refusing exenteration. ${ }^{10}$ Only in cases with non-encapsulated or surgically transected extension did exenteration appear to increase survival. Earlier Starr and Zimmerman had been unable to find evidence that exenteration improved the survival chances of patients with extrascleral extension but recommended increased use of the operation on the grounds that it reduced the likelihood of orbital recurrence and that it was associated with prolonged survival in anecdotal cases. ${ }^{1}$

Although exenteration has a good record in preventing orbital recurrence, Affeldt and coworkers have pointed out that the procedure is infrequently used because of the cosmetic deficit. ${ }^{6}$ While adverse cosmetic factors have probably been overstated, lingering doubts about the value of exenteration in improving survival rate have led to consideration of alternative approaches, particularly for minor degrees of extrascleral extension. Enucleation alone ${ }^{811}$ or followed by radiotherapy ${ }^{6} 1112$ has been recommended for episcleral extension and enucleation plus either tenonectomy or radiotherapy for a well encapsulated extrascleral extension, ${ }^{12}$ with exenteration reserved for a non-encapsulated or surgically transected nodule. ${ }^{7}$ In this centre surgical transection seen only on histopathological examination has not been considered an indication for exenteration, a procedure which has been reserved for individuals with intraoperative evidence of residual orbital melanoma. The rationale for this approach has been that, while radiotherapy is unlikely to achieve sustained local control of bulk disease, it has a good chance of destroying a few cells which may have been left behind in the orbit at operation.

Radiotherapy has not achieved wide acclaim as a treatment of extrascleral extension, mainly because malignant melanoma is a relatively radioresistant tumour. ${ }^{13}$ Nevertheless, sustained tumour regression has been reported by several authors following irradiation of small intraocular melanomas. ${ }^{14-17}$ Only Lommatzsch and Dietrich $^{18}$ and Sobanski and Zeydler ${ }^{19}$ have reported on series in which the orbit has been irradiated after enucleation. Both claim significantly better survival than with enucleation alone. They suggest that postenucleation irradiation damages or destroys tumour cells which may be scattered into the surrounding tissues during the operation to remove the eye, though neither author has presented data on the comparative orbital recurrence rates with and without radiation to support this view.

SURVIVAL

The five-year survival rate of $51 \%$ for patients in the present series compares favourably with the figure of only $34 \%$ alive at five years in Starr and Zimmerman's group of patients with extrascleral extension of uveal melanoma. ${ }^{1}$ However, no direct comparisons can be made between the two groups which are not matched for factors such as tumour size and cell type. Eleven out of the 17 study patients died of melanoma metastases, though 10 did not develop orbital recurrence.

\section{ORBITAL RECURRENCE}

At only $6 \%$ the orbital recurrence rate in the present series is significantly lower than the $18 \%$ reported after extrascleral extension by Starr and Zimmerman,' though, once again, direct comparisons cannot be made because the two groups of patients are not matched. In fact $70 \%$ of the patients in the present series had non-encapsulated or surgically transected melanomas, whereas only $25 \%$ of Starr and Zimmerman's patients had this potentially more serious degree of extrasclera spread. Melanoma may recur in the orbit 20 years or more after enucleation, ${ }^{82021}$ and the incidence of recurrence is unlikely to be fully ascertained without an extremely long period of follow-up. However, both the present series and that of Starr and Zimmerman were followed up for a minimum of five years. The use of radiotherapy after extrascleral extension is not discussed in Starr and

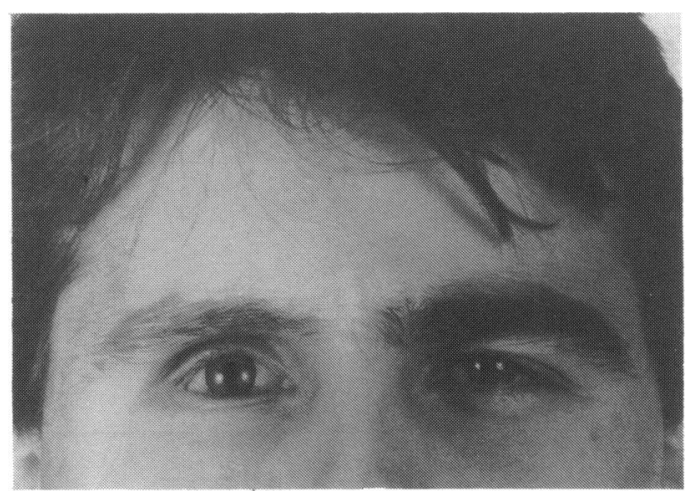

Figure 3: Cosmetic result after radiotherapy with right eye prosthesis in situ. The right eyebrow hair loss is more marked in this patient than is usual. 
Zimmerman's article, though that is not to say that it was not given in some of their patients.

Modern orbital radiotherapy produces very little cosmetic deficit (Fig 3). This is restricted to slight loss of brow hair and thinning of the eyelashes. With adequate fractionation there is very little loss of soft tissue, and tear production is maintained, so that the prosthesis is not dry. Patients receiving radiotherapy can still receive an acrylic orbital implant integrated with the extraocular muscles for realistic movement of the artificial eye. The present study does not produce conclusive evidence of better survival or a reduced orbital recurrence rate when radiotherapy is given after enucleation of an eye with extrascleral extension. A prospective randomised study would be required to settle this issue. Meanwhile the morbidity from orbital radiotherapy is so slight that adjuvant treatment is advised in all cases when extrascleral extension is detected after enucleation for a posterior melanoma except when there is an extensive bulk of residual orbital tumour, when orbital exenteration remains the treatment of choice.

1 Starr HJ, Zimmerman LE. Extrascleral extension and orbital recurrence of malignant melanoma of the choroid and ciliary recurrence of malignant melanoma of the chor
body. Int Ophthalmol Clin 1962; 2: 369-84.

2 Byers WGM, MacMillan JA. Treatment of sarcoma of the uveal tract. Arch Ophthalmol 1935; 14: 967-73.

3 Shammas HF, Blodi FC. Orbital extension of choroidal and ciliary body melanomas. Arch Ophthalmol 1977; 95: 2002-5.

4 Kerschbaumer HP. Das Sarkom des Auges. Weisbaden: Bergmann, 1900 .

5 Jensen OA. Malignant melanomas of the human uvea. Recent follow up of cases in Denmark 1943-1952. Acta Ophthalmol follow up of cases in Den

6 Affeldt DS, Minckler SP, Azen SP, Lin Yeh. Prognosis in uveal melanoma with extrascleral extension. Arch in uveal melanoma with

7 Rendahl I. Does exenteration orbitae improve the prognosis in orbital tumour? Acta Ophthalmol $(K b h)$ 1954; 32: 431-49.

8 Shields JA, Augsberger JJ, Dougherty MJ. Orbital recurrence of choroidal melanoma 20 years after enucleation. $A m \mathcal{F}$ Ophthalmol 1984; 97: 767-70.

9 Kidd MN, Lyness RW, Patterson CC, et al. Prognostic factors in malignant melanoma of the choroid: a retrospective survey of cases occurring in Northern Ireland between 1965 and 1980. Trans Ophthalmol Soc UK 1986; 105: 114-21

10 Kersten RC, Tse DT, Anderson RL, Blodi FC. The role of orbital exenteration in choroidal melanoma with extrascleral extension. Ophthalmology 1985; 92: 436-43.

11 Hungerford J. Current management of choroidal malignant melanoma. Brf Hosp Med 1985; Nov: 287-93.

12 Fraunfelder FT, Boozman FW, Wilson RS, Thomas AH. No touch technique for intraocular malignant melanomas. Arch touch technique for intraocular
Ophthalmol 1977; 95: 1616-20.

13 Reese AB. Tumour of the eye. 3rd ed. New York: Harper and Row, 1976: 198-223.

14 Stallard HB. Radiotherapy for malignant melanoma of the choroid. Brf Ophthalmol 1966; 50: 147-55.

15 Shields JA, Augsberger JJ, Brady LW. Cobalt plaque therapy of posterior uveal melanomas. Ophthalmology 1982;89: 1201-7.

16 Gragoudas ES, Goitein M, Verhey L. Proton beam irradiation of uveal melanoma. Arch Ophthalmol 1982; 100: 928-34.

17 Lommatzsch P. Treatment of choroidal melanomas with Ru-106/Rh-106 beta-ray applicators. Surv Ophthalmol 1974; 19: 85-100.

18 Lommatzsch P, Dietrich B. The effect of orbital irradiation on the survival rate of patients with choroidal malignant melanoma. Ophthalmologica 1976; 173: 49-52.

19 Sobanski J, Zeydler L. Decreased mortality of patients with intranuclear melanoma after enucleation of the eyebal followed by orbital $x$-ray irradiation. Polish Med F 1972; 11 1512-6.

20 Saunders DH, Rodrigues MM, Shannon GM. Orbital recurrence of malignant melanoma of the choroid 24 years after enucleation. Ophthalmic Surg 1977; 8: 31-3.

21 Allen JC, Jaeschke WH, Madison W. Recurrence of malignan melanoma in an orbit after 28 years. Arch Ophthalmol 1966 76: 79-81 